\title{
Prevalence of brucellosis in dairy cattle from the main dairy farming regions of Eritrea
}

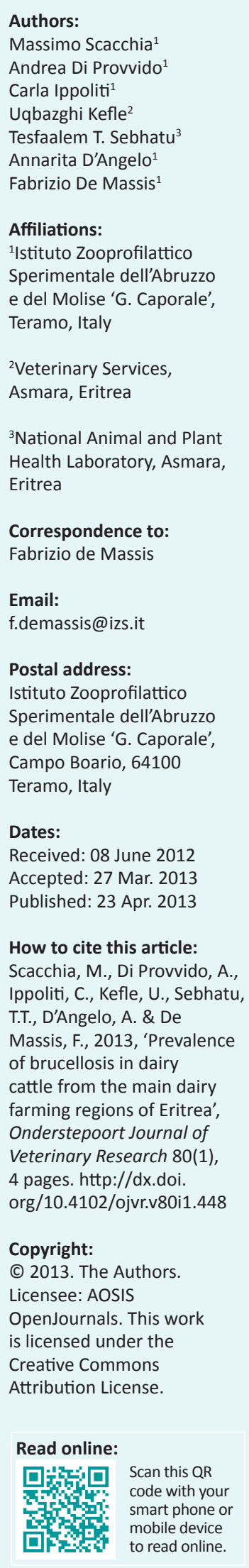

In order to get a reliable estimate of brucellosis prevalence in Eritrean dairy cattle, a crosssectional study was carried out in 2009. The survey considered the sub-population of dairy cattle reared in modern small- and medium-sized farms. Samples were screened with the Rose Bengal test (RBT) and positive cases were confirmed with the complement fixation test (CFT). A total of 2.77\% (417/15 049; Credibility Interval CI: 2.52\% - 3.05\%) of the animals tested in this study were positive for antibodies to Brucella species, with a variable and generally low distribution of positive animals at regional level. The highest seroprevalence was found in the Maekel region (5.15\%; CI: $4.58 \%-5.80 \%)$, followed by the Debub (1.99\%; CI: $1.59 \%-$ $2.50 \%)$ and Gash-Barka (1.71\%; CI: $1.34 \%-2.20 \%)$ regions. Seroprevalence at sub-regional levels was also generally low, except for two sub-regions of Debub and the sub-region Haicota from the Gash-Barka region. Seroprevalence was high and more uniformly distributed in the Maekel region, namely in the Asmara, Berik and Serejeka sub-regions. Considering the overall low brucellosis prevalence in the country, as identified by the present study, a brucellosis eradication programme for dairy farms using a test-and-slaughter policy would be possible. However, to encourage the voluntary participation of farmers to the programme and to raise their awareness of the risks related to the disease for animals and humans, an extensive public awareness campaign should be carefully considered, as well as strict and mandatory dairy movement control.

\section{Introduction}

Brucellosis is one of the most common zoonotic diseases in the world. The geographical distribution of brucellosis constantly changes as new foci emerge or re-emerge. The disease occurs worldwide in both animals and humans, except in those countries where bovine brucellosis has been eradicated. The worldwide economic losses due to brucellosis are extensive, not only in terms of animal production but also in terms of human health. However, when the incidence of brucellosis is controlled in the animal reservoirs, there is a corresponding and significant decline in the incidence in humans (Seleem, Boyle \& Sriranganathan 2010). In Eritrea, brucellosis is an important disease in both animals and humans; most human cases appear to be related to the consumption of unpasteurised milk (Kebedom et al. 2008). Literature shows that there have been some seroprevalence studies carried out in domestic animals (Omer et al. 2000a, 2000b) and humans (Omer et al. 2002). However, they were limited both in terms of number of animals tested and their geographical extent. Knowledge about brucellosis prevalence in a country is of the utmost importance in order to set up a strategy to control and eradicate the disease. Therefore, in 2009, the Ministry of Agriculture carried out a new serological survey for brucellosis in Eritrea. The objective was to estimate the seroprevalence of brucellosis in dairy cattle in Eritrea, in order to identify strategies for control and eradication of the disease in the country.

\section{Material and Methods}

\section{Study areas}

Eritrea is divided into six administrative regions: Maekel, Debub, Anseba, Gash-Barka, Northern Red Sea and Southern Red Sea. The study was carried out in the first five regions, where dairy farming is practiced. Southern Red Sea was excluded because there are limited dairy activities due to the dry climate. The population of dairy cattle reared in modern small and medium-sized farms were included in the study. These farms are mainly located in the urban and peri-urban areas in all of the five regions selected for the study.

\section{Animals}

The dairy cattle population in Eritrea is estimated to be around 28000 and is comprised of three types of breeds: Holstein Friesian (90\%); indigenous breeds, mainly Barka (approximately $8 \%-9 \%$ ); and crossbreeds of Holstein Friesian and Barka (approximately 1\% - 2\%). The three main 
cattle husbandry systems are: the intensive urban and periurban system (mainly devoted to dairy cattle); the extensive mixed crop-livestock system used in the southern highlands; and the extensive (traditional) pastoral husbandry system used in the western and eastern lowlands. The serological study included all sexually mature dairy cattle (both male and female) present in individual dairy farms in urban and peri-urban areas and was planned and coordinated by the Ministry of Agriculture of Eritrea during 2009. There is no vaccination against animal brucellosis in Eritrea, and thus all samples were from unvaccinated animals.

\section{Sample collection}

Sera were collected in $10 \mathrm{~mL}$ sterile tubes by jugular venipuncture. Sera were stored in refrigerators $\left(0^{\circ} \mathrm{C}-4^{\circ} \mathrm{C}\right)$ and transported in iceboxes to the Central Veterinary Laboratory in Asmara. Regional veterinary teams established for this purpose collected the samples.

\section{Sample testing}

All the tests were carried out at the Central Veterinary Laboratory in Asmara. Serum samples were tested for Brucella antibodies using the Rose Bengal test (RBT) as a screening test and all positive reactors were confirmed with the complement fixation test (CFT). Both tests were performed according to the provisions of the World Organization for Animal Health (OIE) Manual of Diagnostic Tests and Vaccines for Terrestrial Animals (World Organization for Animal Health (OIE) 2008). To increase the specificity of test results, only samples that tested positive on both RBT and CFT were considered to be positive for Brucella.

\section{Statistical analysis}

Results obtained in this study were estimated and compared using a Bayesian approach (Sivia 1996). This is an application of the Bayes theorem (Bayes 1763), which allows the investigator to integrate any previous knowledge (expressed as a prior probability distribution), with the likelihood of obtaining a certain result if the animals are infected (likelihood functions), with the results obtained by the application of the tests to a given population (collected data). The final results are probability distributions of the proportion of infected animals in the population (posterior probability). Probabilities of the various possible prevalence values were estimated using a binomial likelihood function and an uninformed Uniform $(0,1)$ prior distribution. As existing knowledge on the prevalence of infected animals was considered to be virtually zero, an uninformed Uniform $(0,1)$ prior distribution was used. The Uniform $(0,1)$ distribution states that prior to the collection of data, all true probability values are considered possible within the range defined for the number of infected animals (prevalence calculation at animal level). The Upper (UCL) and Lower (LCL) 95\% credibility levels (limits of the 95\% Credibility Interval [CI]) were calculated using a beta probability distribution (Vose 2000). The primary advantage of using a Bayesian method over a traditional statistical method is that uncertainty in each parameter is modelled as a probability distribution, thus, the posterior distributions that are obtained in the analysis can be used to calculate the $95 \%$ CI for prevalence. In contrast to confidence intervals, credibility intervals have a direct probabilistic interpretation (Wagner et al. 2003). Calculations were performed using MS-Excel ${ }^{\circledR}$ for Windows $^{\circledR}$, version 2003 (MS Excel 2003).

\section{Results}

The total number of animals sampled from the different regions was 15049 . A total of $2.77 \%$ (CI: $2.52 \%-3.05 \%$ ) tested positive to antibodies to Brucella species (Table 1).

The geographical distribution of test results at regional level is shown in Figure 1. The distribution of positive animals at regional level is variable and generally low. The highest seroprevalence was found in the Maekel region (5.15\%; CI: $4.58 \%-5.80 \%)$, followed by the Debub (1.99\%; CI: $1.59 \%-$ $2.50 \%)$ and Gash-Barka (1.71\%; CI: $1.34 \%-2.20 \%)$ regions. In the Anseba and Northern Red Sea regions, seroprevalences were $0.70 \%$ (CI: $0.43 \%-1.15 \%$ ) and $1.47 \%$ (CI: $0.81 \%-2.69 \%)$ respectively (see Table 1 and Figure 1). As far as the Maekel region is concerned, the LCL of the percentage of positive animals is higher than the UCL of the percentage of positive animals identified in the other regions. Therefore, the seroprevalence recorded in the Maekel region is significantly higher than all of the other regions investigated. As far as the Anseba region is concerned, the UCL of the percentage of positive animals is lower than the LCL of the percentage of positive animals identified in the Maekel, Debub and GashBarka regions. Therefore, the seroprevalence recorded in the Anseba region is significantly lower than the ones recorded in the Maekel, Debub and Gash-Barka regions.

\section{Brucellosis at sub-regional level}

Seroprevalence at sub-regional levels (Figure 2) is generally low, except for three sub-regions in the Maekel region

TABLE 1: Test results for brucellosis in dairy cattle at regional level in Eritrea, 2009.

\begin{tabular}{llllll}
\hline Region & $\begin{array}{l}\text { Number of tested } \\
\text { animals }\end{array}$ & $\begin{array}{l}\text { Number of positive-tested } \\
\text { animals }\end{array}$ & \% Positive & $\begin{array}{l}\text { \% Upper Credibility } \\
\text { Level }\end{array}$ & $\begin{array}{l}\text { \% Lower Credibility } \\
\text { Level }\end{array}$ \\
\hline Maekel & 5005 & 258 & 5.15 & 5.80 & 4.58 \\
Debub & 3663 & 73 & 1.99 & 2.50 & 1.59 \\
Gash-Barka & 3556 & 61 & 1.72 & 2.20 & 1.15 \\
Anseba & 2147 & 15 & 0.70 & 2.69 & 0.43 \\
Northern Red Sea & 678 & 10 & 1.47 & $\mathbf{3 . 0 5}$ & $\mathbf{2 . 7 7}$ \\
\hline Total & $\mathbf{1 5 0 4 9}$ & $\mathbf{4 1 7}$ & $\mathbf{2 . 5 2}$ & \\
\hline
\end{tabular}

Source: Ministry of Agriculture, Eritrea 
(Asmara, Berik and Serejeka), two sub-regions in the Debub region (Adikeih and Adiquala) and the sub-region of Haikota in the Gash-Barka region. However, the number of infected animals in the sub-regions of Adikeih, Adiquala and Haicota is small (22, 17 and 10 respectively).

\section{Discussion}

The results of this study show that the percentage of dairy cattle positive for Brucella antibodies in RBT and CFT in Eritrea is generally low. This excludes the Maekel region (Figure 1), which shows an overall percentage slightly above $5 \%$ (Table 1). The seroprevalence recorded in the Maekel region is significantly higher than the other regions. This is in contrast to the seroprevalence recorded in the Anseba region, which is significantly lower than those recorded in the Maekel, Debub and Gash-Barka regions. This suggests that further investigation is needed to assess the possible

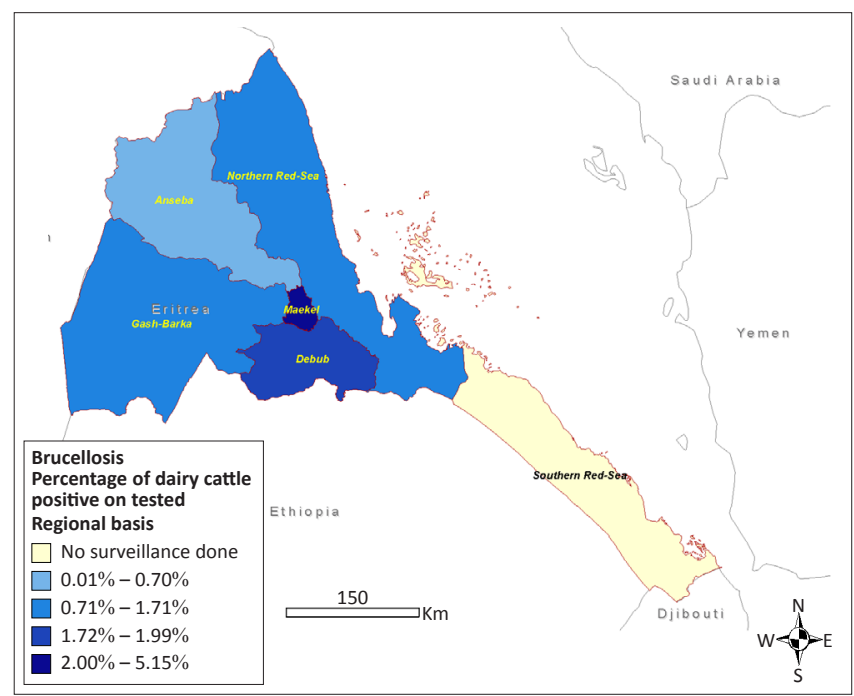

FIGURE 1: Geographical distribution of test results for brucellosis in dairy cattle in Eritrea at regional level in 2009.

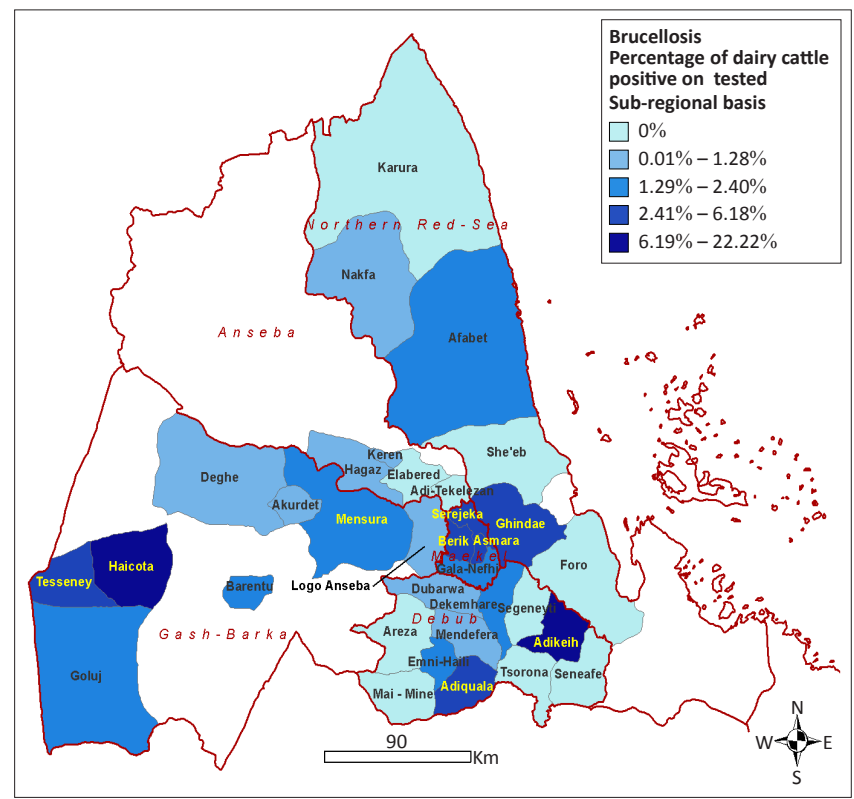

FIGURE 2: Geographical distribution of test results for brucellosis in dairy cattle in Eritrea at sub-regional level in 2009. causes of the observed variation of brucellosis prevalence in the different regions. This is confirmed by the fact that at sub-regional level the seroprevalence is generally low (Figure 2), (except for two sub-regions in Debub and the subregion Haicota from the Gash-Barka region) and the number of infected animals in these sub-regions is small (only 22, 17 and 10, in Adikeih, Adiquala and Haicota sub-regions, respectively).

Studies investigating brucellosis in dairy cattle have been scarce in the area concerned. Omer et al. (2000) found an individual seroprevalence of $8.2 \%$ in dairy herds kept under the intensive husbandry systems of the farms around the capital, Asmara. Higher percentages of brucellosis-positive dairy cattle were obtained in a study carried out by Omer et al. (2007) in the Kassala Area, Eastern Sudan, near to the Eritrean border. This study was conducted during 2004- 2006 and identified a mean of $10.9 \%$ of cattle testing positive. However, this figure could have been overestimated due to the low number of cattle tested during the period $(n=694)$.

Losses due to brucellosis are extensive, not only in terms of animal production, but also in human health. However, when the incidence of brucellosis is controlled in the animal reservoirs, there is a corresponding and significant decline in the incidence in humans De Massis et al. (2005). It is hoped that the results of the present study will encourage the start of a brucellosis-eradication programme using the test-and-slaughter policy in Eritrea. The number of seropositive animals is low and costs of slaughtering could be handled either by the owners themselves or through partial or total compensation from the dairy associations or the Government. The latter strategy would greatly help the owners in restocking animals and affording the indirect economic losses related to the presence of the disease in herds.

However, one of the keys to a successful eradication programme is the voluntary participation of the animal owners. To reach this objective it is important to increase the public awareness about the risks related to the disease in animals and human beings. This requires an increase in the knowledge of disease control and eradication by the veterinary services, which would increase the trust of farmers in the system in order to guarantee their complete and voluntary participation in the eradication campaign. Moreover, to complement and support the eradication programme, the participation of the farmers should be encouraged through establishing some market-reward system (i.e. easy market accesses to farmers with brucellosisfree herds and restrictions to access the milk markets for owners with seropositive herds).

\section{Conclusions}

The percentage of dairy cattle positive for Brucella antibodies in RBT and CFT in Eritrea is generally low, except for the Maekel region (see Figure 1), which shows an overall percentage slightly above $5 \%$. Considering the overall low 
brucellosis prevalence in Eritrea, a brucellosis-eradication programme for dairy cattle herds through a test-andslaughter policy could be considered. However, to encourage voluntary farmer participation and to raise awareness about the risks related to the disease in animals and human beings, an extensive public awareness campaign is vital, as well as strict and mandatory dairy movement control.

\section{Acknowledgements}

Activities were carried out in the framework of the OIE Twinning Project on Brucellosis between the National Veterinary Laboratory of Eritrea and the Istituto Zooprofilattico Sperimentale dell'Abruzzo e del Molise 'G. Caporale' in Teramo, Italy.

\section{Competing interests}

The authors declare that they have no financial or personal relationship(s) that may have inappropriately influenced them in writing this article.

\section{Authors' contributions}

M.S. (Istituto Zooprofilattico Sperimentale dell'Abruzzo e del Molise 'G. Caporale') was the project leader. M.S. and A.D.P. (Istituto Zooprofilattico Sperimentale dell'Abruzzo e del Molise 'G. Caporale') were both responsible for the project design. C.I. (Istituto Zooprofilattico Sperimentale dell'Abruzzo e del Molise 'G. Caporale') assisted in data calculations and map production. U.K. (National Animal and Plant Health Laboratory) made conceptual contributions and carried out the literature review. T.T.S. (National Animal and Plant Health Laboratory) and A.D'A. (Istituto Zooprofilattico Sperimentale dell'Abruzzo e del Molise 'G. Caporale') made conceptual contributions. F.D.M.. (Istituto Zooprofilattico Sperimentale dell'Abruzzo e del Molise 'G. Caporale') carried out data analysis and wrote the manuscript.

\section{References}

Bayes, T., 1763, 'An essay towards solving a problem in the doctrine of chances', Philosophical Transactions of the Royal Society of London 53, 370-418 viewed 01 December 2011, from www.stat.ucla.edu/history/essay.pdf

De Massis, F., Di Girolamo, A., Petrini, A., Pizzigallo, E., \& Giovannini, A., 2005, Correlation between animal and human brucellosis in Italy during the period 1997-2002. Clinical Microbiology and Infection 11, 632-636. http://dx.doi. org/10.1111/j.1469-0691.2005.01204.x, PMid:16008615

Kebedom, A., Teclebirhan, T., Tekest, T., Zekarias, B., Mufunda, J. \& Gebremichael, A., 2008, 'Recent lessons learnt from the outbreak of brucellosis in Dekemhare, Anseba, Eritrea', Journal of the Eritrean Medical Association 3, 19-21, viewed 01 December 2011, from http://www.ajol.info/index.php/jema/article/view/49622

MS Excel, 2003, Computer software, Microsoft Windows, Redmond, Washington.

Omer, M.K., Assefaw, T., Skjerve, E., Tekleghiorghis, T. \& Woldehiwet, Z., 2002 'Prevalence of antibodies to Brucella spp. and risk factors related to high-risk 'Prevalence of antibodies to Brucella spp. and risk factors related to high-risk
occupational groups in Eritrea', Epidemiology and Infection 129, 85-91. http:// occupational groups in Eritrea', Epidemiology and Infection 129, 85-91. htp:/
dx.doi.org/10.1017/S0950268802007215, PMid:12211600, PMCid:2869878

Omer, M.K., Skjerve, E., Holstad, G., Woldehiwet, Z. \& Macmillan, A.P., 2000a, 'Prevalence of antibodies to Brucella spp. in cattle, sheep, goats, horses and camels in the State of Eritrea; influence of husbandry systems', Epidemiology and Infection 125, 447-453. http://dx.doi.org/10.1017/S0950268899004501 PMid:11117970, PMCid:2869619

Omer, M.K., Skjerve, E., Woldehiwet, Z. \& Holstad G., 2000b, 'Risk factors for Brucella spp. infection in dairy cattle farms in Asmara, State of Eritrea', Preventive Veterinary Medicine 46, 257-265. http://dx.doi.org/10.1016/S0167-5877(00)00152-5

Omer, M.M., Abdelaziz, A.A., Abusalab, S.M.A. \& Ahmed, A.M., 2007, 'Survey of Brucellosis among Sheep, Goats, Camels and Cattle in Kassala Area, Eastern Sudan', Journal of Animal and Veterinary Advances 6, 635-637.

Seleem, M.N., Boyle, S.M. \& Sriranganathan, N., 2010, 'Brucellosis: A re-emerging zoonosis', Veterinary microbiology 140, 392-398. http://dx.doi.org/10.1016/j. vetmic.2009.06.021, PMid:19604656

Sivia, D.S., 1996, Data analysis. A Bayesian tutorial, Clarendon, Oxford.

Vose, D., 2000, Risk analysis: A quantitative guide, 2nd edn., John Wiley \& Sons, Chichester.

Wagner, B., Gardner, I., Cameron, A., Doherr, M.G., 2003, 'Statistical analysis of data from surveys, monitoring, and surveillance systems', in M.D. Salman (ed.), Anima Disease Surveillance and Survey Systems: Methods and Applications, Blackwell publishing, Ames, lowa. http://dx.doi.org/10.1002/9780470344866.ch5

World Organization for Animal Health (OIE), 2008, Manual of Diagnostic Tests and Vaccines for Terrestrial Animals, viewed 01 December 2011, from http://www.oie. int/international-standard-setting/terrestrial-manual/access-online/ 